\title{
Tecnologías de la Información y Comunicación: \\ Una estrategia para fortalecer la \\ formación en educación ambiental ${ }^{1}$
}

\section{Information and Communication Technologies: A strategy to strengthen training in environmental education}

DOI: http://dx.doi.org/10.17981/cultedusoc.9.3.2018.78

Artículo de investigación. Fecha de recepción: 15/06/2018. Fecha de aceptación: 27/11/2018

William Quintero Mandón";

Fabrican Castrillo De la Rosa; José Gregoria Lara;

Yimena Perdomo Solano; William Quintero Mandón;

José Villegas Ortiz y Juan Vizcaino Fontalvo ${ }^{3}$

IE Manuel Salvador Meza Camargo, Municipio Sabana de San Ángel Magdalena (Colombia) wq1789@gmail.com

Para citar este artículo:

Quintero, M., Castrillo, F., Lara, J., Perdomo, Y., Quintero, W., Villegas, J. y Vizcaino, J. (2018). Tecnologías de la Información y Comunicación: Una estrategia para fortalecer la formación en educación ambiental. Cultura. Educación y Sociedad 9(3), 661-666. DOI: http:// dx.doi.org/10.17981/cultedusoc.9.3.2018.78

\section{Resumen}

El presente artículo muestra los resultados de las Tecnologías de la Información y la Comunicación (TIC), en su amplio uso en la actualidad, ofrece una alternativa importante para aplicar en el área educativa. El presente proyecto de investigación tiene como propósito fortalecer los procesos educativos a través de estrategias pedagógicas para el fortalecimiento de la educación ambiental, desde el uso de las TIC, en la Institución Educativa Departamental Manuel Salvador Meza Camargo del Municipio de Sabana de San Ángel Magdalena, mediante la implementación de talleres lúdicos-pedagógicos, en donde se permitan adquirir destrezas, habilidades y competencias en los diferentes ámbitos, promoviendo la participación activa en las actividades lideradas por los diferentes actores que hacen parte de la comunidad educativa. Se logró identificar que los estudiantes tienen conocimientos acerca de la temática; sin embargo, es fundamental desarrollar estrategias para el fortalecimiento y permanencia de la educación ambiental en la institución.

Palabras clave: Tecnología de la información y la comunicación, estrategias pedagógica, educación ambiental.

\section{Abstract}

This article shows the results of the Information and Communication Technologies (ICT), in its wide use at present, it offers an important alternative to apply in the educational area. The purpose of this research project is to strengthen educational processes through pedagogical strategies for the strengthening of environmental education through the use of ICT in the Manuel Salvador Meza Camargo Departmental Educational Institution of the Municipality of Sabana de San Ángel Magdalena, through the implementation of playful-pedagogical workshops, which allow acquiring skills, abilities and competences in different areas, leading to active participation in activities led by the different actors that are part of the educational community. It was possible to identify that students have knowledge about the subject, however, it is essential to develop strategies for the strengthening and permanence of environmental education in the institution.

Keywords: Information and communication technology, pedagogical strategies, environmental education.

1 Este artículo ha sido derivado del Proyecto Fortalecimiento de la Cultura Ciudadana y Democrática en CT+I a través de la IEP apoyada en TIC en el Dpto. del Magdalena.

2 Líder del grupo de investigación "BICICLETEANDO ANDO".

3 Docentes de la institución Educativa Manuel Salvador Meza Camargo del Municipio de Sabana de San Ángel Magdalena .

- The author; licensee Universidad de la Costa - CUC

Cultura, Educación y Sociedad vol. 9 no. 3, pp. 661-666. Diciembre, 2018

Barranquilla. ISSN 2389-7724 Online 


\section{Introducción}

Desde lo plasmado en la Carta de Belgrado (1975), respecto a la educación como medio para fomentar y afianzar la conciencia del cuidado del ambiente, ha habido múltiples acuerdos y orientaciones sobre la importancia de trabajar este tema en las instituciones educativas. Una directriz que resalta es: la educación tiene un rol muy importante como herramienta para lograr procesos de cambio social, es necesario mejorar los procesos de enseñanza con nuevos enfoques teóricos y prácticos, con la intención de sembrar un cúmulo de valores y actitudes de compromiso y ética ambiental.

Partiendo de lo anterior, el presente proyecto de investigación tiene como propósito fortalecer los procesos educativos a través de estrategias pedagógicas para el mejoramiento de la educación ambiental, desde el uso de las Tecnologías de la Información y la Comunicación (TIC), en la Institución Educativa Departamental (IED), Manuel Salvador Meza Camargo, del Municipio de Sabanas de San Ángel, Magdalena.

Para cumplir ese objetivo, se desarrolló la implementación de talleres lúdicos-pedagógicos, respaldados por la utilización de las TICS, dirigidos a fortalecer y potencializar destrezas, habilidades y competencias en los diferentes ámbitos en que se desenvuelve el estudiante.

El grupo de investigación está formado por 6 docentes de la Institución Educativa Departamental (IED) Manuel Salvador Meza Camargo; docentes - maestros acompañantes, coinvestigadores y maestros que introducen la Investigación como Estrategia Pedagógica (IEP), al currículo de la sede Manuel Salvador Meza.

El desarrollo de este proyecto cuenta con la participación activa de los docentes, que lideran las actividades por medio de estrategias pedagógicas dirigidas a mantener la motivación en los estudiantes. Bravo (2008) señala que las estrategias o prácticas pe- dagógicas son las acciones previstas por el docente, con el fin de facilitar la formación y el aprendizaje de los estudiantes, son actividades formativas y de interacción para desarrollar conocimientos, valores, prácticas, procedimientos y problemas propios del campo de formación. Atendiendo a lo que pretende este proyecto investigativo, el centro de este estudio son las estrategias pedagógicas, las cuales juegan un papel fundamental en la captación y atención a los estudiantes, para promover un impacto significativo en la interiorización del fomento de la educación ambiental (Franco, 2012; Fernández, Luke, Leal, 2010).

A nivel nacional, se encuentran diversas investigaciones relacionadas con el fortalecimiento de la educación ambiental, teniendo en cuenta el uso de la TICS como herramienta básica para mejorar y ampliar todos los procesos escolares del educando, e implementando espacios de reflexión y potenciando el aprendizaje colaborativo. (Monsalve, 2011; Fajardo, 2007; Hinostroza, 2004).

Los estudiantes, con el uso de las TICS desarrollan independencia en sus procesos; además, a partir de su buen uso, se pueden propiciar espacios personales de reflexión que lo llevan a la autorregulación, promoviendo el desarrollo integral de su personalidad, la autonomía e independencia y la capacidad de crecer y aprender en su vida.

En este sentido, según Martínez-Otero y Ojeda (2011), las TICS no solo propician un mejor rendimiento en el educando, sino que con un uso orientado podrían potencializar aspectos cognitivos y destrezas importantes en los estudiantes.

La Institución Educativa Departamental Manuel Salvador Meza, ubicada en sabana de San Ángel, zonal rural del Magdalena, presenta dificultades respecto a los entornos en que se desenvuelven los estudiantes, los espacios no son adecuados para la escolarización, esto ha generado poca conciencia ambiental en los estudiantes, 
quienes terminan aportando con el poco cuidado ambiental de esta zona.

Por esta razón se hace necesario la ejecución del presente proyecto investigativo, debido a que permite fortalecer actitudes, valores y compromisos frente al cuidado del medio ambiente, el cual debe entenderse en su sentido dinámico, esto es, el conjunto de actividades humanas tendientes a evitar la afectación, alteración o puesta en riesgo la existencia de la vida misma (Pereira, 2016); lo anterior, a través del uso de las herramientas tecnológicas, lo cual contribuye al enriquecimiento e innovación de estrategias pedagógicas que permiten al estudiante mejorar el proceso de aprendizaje integral.

\section{Metodología}

El artículo que se presenta es un producto derivado del estudio de la Tecnologías de la Información y Comunicación: Una estrategia para fortalecer la formación de la educación ambiental. Desarrollado en la Institución Educativa Departamental (IED) Manuel Salvador Meza Camargo; el estudio contempla un procedimiento metodológico como sigue:

En cuanto al diseño metodológico se utilizó un enfoque cualitativo (Blasco y Pérez, 2007; Pérez, 2002; Hernández 2014), porque estudia la realidad en su contexto natural y como sucede, sacando e interpretando fenómenos con la participación de las personas implicadas. Se abordó el modelo de acción participativa, que es una propuesta metodológica que involucra a la comunidad en el conocimiento y solución de sus problemas. En este sentido, frente a la pertinencia y relevancia de esta investigación, no busca solamente describir los problemas, sino generar con la comunidad los conocimientos requeridos para definir las acciones pertinentes al cambio y la transformación que se requiera (Schutter, 1987; Báez, 2009; Pérez-Acosta \& Urrá, 2015).
El alcance de la investigación es descriptivo porque desarrolla de modo sistemático las características de una situación o área de interés, además de los acontecimientos actuales de los padres de familias y/o cuidadores de los estudiantes de la institución educativa.

Los instrumentos utilizados fueron la observación, la entrevista estructurada, la cual Rodríguez (2012), la define como el "conjunto de procedimientos en el que el entrevistador utiliza preguntas dirigidas a un abordaje detallado, ajustado a ciertos objetivos preestablecidos que desea conocer o para los cuales el entrevistado solicita asistencia”.

Las unidades de análisis fueron seleccionadas intencionalmente, contando con 19 estudiantes de la institución educativa Manuel Salvador Meza. Se identificó que los estudiantes tienen conocimientos acerca de la temática, sin embargo, es fundamental desarrollar estrategias para el fortalecimiento y permanencia de la educación ambiental en la institución.

\section{Resultados}

Se presentan lo hallazgos derivados del instrumento entrevista estructurada, construido para el desarrollo del proyecto de investigación. Para estos hallazgos se tuvo en cuenta la pregunta orientadora. ¿De qué manera la estrategia pedagógica fortalece la educación ambiental desde el uso de las TIC en la Institución Educativa Departamental Manuel Salvador Meza Camargo? Ante esta interrogante, se generaron tres planteamientos;

- Los estudiantes perciben los recursos naturales a través del agua, los árboles y el suelo.

- Dirigida está a cómo hacer conciencia sobre la educación ambiental, los estudiantes proponen concientizar la educación ambiental a través de campañas pedagógicas- reflexivas que generen una conciencia ambiental, técnica pedagógica. 
- Los estudiantes, resaltan la importancia de la ejecución de actividades pedagógicas formativas que contribuya a la calidad de vida en los diferentes contextos.

\section{Conclusiones}

Considerando la importancia de la preservación del medio ambiente, es fundamental hacer una transversalidad de este tema en los currículos académicos de todas las instituciones educativas, ya que esto permitirá generar conciencia en los estudiantes sobre la necesidad de dar un uso sostenible a los recursos naturales. Además, entendiendo esto como una responsabilidad de todos los seres humanos, lo que se realice hoy con la naturaleza traerá consecuencias del mismo origen en un tiempo futuro.

Hoy en día observamos que a nivel mundial el medio ambiente está presentando problemas para poder conservar su equilibrio, ha habido diversos daños sufridos en el planeta, los cuales han sido a causa de la industrialización y las explosiones demográficas incorrectas. Por lo cual, para poder conseguir que el ser humano logre aprender acerca de la importancia de la conservación del medio ambiente, se hace necesario enseñar ciertos valores de respeto y cuidado desde que el individuo están en las instituciones educativas, es por esto que materias como lo es la ecología o el reciclaje son fundamentales en la educación de los niños para que posteriormente esta salvaguardia se haga existente.

El uso de las TICS, que en la actualidad es universal, es una herramienta valiosa, que permite difundir información, formar personas, conocer aspectos relevantes de diferentes temáticas, y en general su uso es muy amplio. En este sentido, autores como, Betancur, Carmona, Contreras, Karam, Maestre, Romero y Uribe (2014) y Marín, Niebles, Sarmiento y Valbuena, S. (2017), destacan la importancia de la mediación de las tecnologías, específicamente la internet en el ámbito didáctico por cuanto fortalece las interacciones y la configuración de redes o entornos virtuales de aprendizaje. Su utilización como herramienta para orientar a una mejora educativa, logra contextualizar a la educación al nivel en que se encuentra la tecnología y así mismo estar a la vanguardia con temas tan relevantes como lo es el cuidado del medio ambiente.

Por lo tanto, es indispensable que las tecnologías de la información y comunicación (TIC) y todos los funcionarios, tanto educativos como gubernamentales, esten implicados en el desarrollo, organización, utilización responsable y sostenibilidad de los recursos, y así poder patrocinar las decisiones necesarias para mitigar el impacto negativo que se generan en el medio ambiente; aprovechando los aportes educativos y la información disponible mediante las TIC, bien sea por Internet y demás medios. Las TIC son importantes cuando se trata de advertir sobre los catástrofes naturales que pueden surgir como secuela del cambio climático y permiten el desarrollo de mecanismos humanitarios de ayuda que se requiera.

Con los resultados hallados en esta investigación, se logra comprender que es completamente útil utilizar los medios tecnológicos para dotar de herramientas a los estudiantes, que les permitan afianzar conocimientos en el cuidado del medio ambiente, que genere en ellos una cultura sostenible y que perdure en el tiempo, pasando de generación en generación para contribuir a mejorar el mundo en el que viven. La Educación Ambiental, hoy más denominada Educación para la Sostenibilidad o Educación Ambiental para el Desarrollo Sostenible, debe potenciar el manejo de conceptos de una forma constructivista, significativa y permanente, el compromiso con valores, principios, actitudes y comportamientos que permitan la comprensión del contexto y la naturaleza en la que se vive. 
La educación ambiental sustentada en las TICS genera ciertas ventajas frente a la educación habitual, debido a que ofrece múltiples factores como son la disponibilidad de computadores y celulares en los hogares e instituciones educativas, el acceso a internet, el deseo y dominio de la mayoría de las estudiantes por la tecnología, la propagación de mensajes de texto, e implementación de las estrategias pedagógicas acordes con el modelo de Educación Ambiental. En este orden de ideas, en Colombia es indispensable propiciar investigaciones en este tema y seguir impulsando un modelo pedagógico para una educación ambiental, dinámica y participativa que facilite el desarrollo de una cultura ambiental. El uso de las TIC en educación ambiental desarrolla vínculos entre docentes y estudiantes, fortaleciendo sus relaciones y rompiendo la barrera espacio- tiempo.

Las TIC benefician el mejoramiento de algunas destrezas y habilidades, muchas de ellas difíciles de lograr con el sistema educativo tradicional. Es por esto que en las instituciones se enseñan las habilidades básicas como lo son el navegar, buscar, seleccionar, organizar y manejar conceptos y/o información que puedan ayudar tanto a docentes como a estudiantes en su formación. La tecnología, a diferencia del sistema convencional que se basa en la palabra, el tablero y el texto, proporciona herramientas y competencias investigativas tales como navegar y dar una mirada por el mundo real o virtual, adquiera nuevas dimensiones del mismo, desarrolle su conocimiento sobre aportes científicos y de su entorno social.

El uso de estas tecnologías ofrece la oportunidad al estudiante para aprender sin barreras, puesto a que las imágenes y los videos le permiten conocer diferentes ambientes, construyendo así el conocimiento de una manera en la que resultaría inaplicable si se utilizan fuentes de información tradicionales.
El estudio de estos ambientes favorece la habilidad de los estudiantes para investigar y enriquecer sus propios conocimientos al indagar en nuevas fuentes de información. En consecuencia, la Educación Ambiental requiere y propicia la utilización de diversos recursos, como ilustraciones y videos que fomenten el aprendizaje significativo de aspectos ambientales, lo cual podría estar oculto con tan sólo leer un simple texto. Estos recursos puedan estimular la transformación de las estudiantes, de seres pasivos a participantes predominantemente activos en su propio proceso de aprendizaje en la educación ambiental.

\section{Referencias}

Báez, J. (2009). Investigación cualitativa. Madrid: ESIC Editorial.

Betancur, S., Carmona, L., Contreras, R., Karam, J., Maestre, N., Romero, Y. y Uribe, S. (2014). Videojuegos y tic como Estrategias Pedagógicas: Formación para el uso seguro de internet. Cultura Educación y Sociedad, 5(1). Recuperado de https://revistascientificas.cuc.edu. co/culturaeducacionysociedad/article/ view/997

Blasco, M. y Pérez, J. (2007). Metodologías de investigación en las ciencias de la actividad física y el deporte. Sant Vicente (Alicante): Club Universitario.

Bravo, H. (2008). Estrategias pedagógicas. Córdoba: Universidad del Sinú

Fajardo, J.. (2007). Desafíos de la educación. Recuperado de http://www.plandecenal. edu.co/html/1726/w3-article-126746. html

Fernández, O., Luquez, P. y Leal, E. (2010) Procesos socio-afectivos asociados al aprendizaje y práctica de valores, en el ámbito escolar. Telos, 12(1). 63-78. Disponible en http://redalyc.uaemex.mx/ s r c / i n i c i o / A r t P d f R e d. jsp?iCve=99312518005. 
Franco, S. (2012). La educación como Praxis Transformadora. Universidad Pedagógica Nacional, 36. 45-56

Hernández, R., Fernández, C. y Baptista, P.(2014).Metodologíadelainvestigación. México, D.F.: McGraw-Hill Education.

Hinostroza, J. (2004). Diseño de estrategias de innovación y TIC para el desarrollo de la educación. Innovar en la enseñanza y enseñar a innovar. Recuperado de http://www.expansiva.cl/media/en_foco/ documentos/05052004211607.pdf.

Marín, F., Niebles, M., Sarmiento, M. y Valbuena, S. (2017): Mediación de las tecnologías de la información en la comprensión lectora para la resolución de problemas aritméticos de enunciado verbal. Espacios, 38(20) Recuperado de http://www.revistaespacios.com/ a17v38n20/a17v38n20p20.pdf

Martínez-Otero, V. y Ojeda, G. (2011). Análisis pedagógico de plataformas audiovisuales mediante el modelo pentadimensional del discurso educativo. Revista Iberoamericana de Educación, 57. 167-181. Recuperado de https://dialn e t . u n i r i oj a.es/s e r v le t/ articulo?codigo $=4770798$

Monsalve, M. (2011). Implementación de las tics como estrategia didáctica para generar un aprendizaje significativo de los procesos celulares en los estudiantes de grado sexto de la institución educativa San Andrés del Municipio de Girardota. [Trabajo final]. Universidad Nacional de Colombia, Medellín. Recuperado de http://www.bdigital.unal.edu. co/5936/1/43666105.2012.pdf
Patiño, A. (2015). Tendencias tecnológicas que influyen en el aumento de la productividad empresarial. INGE CUC, 11(2), 84-96. https://doi.org/10.17981/ingecuc. 11.2.2015.09

Pereira, M. (2016). Hacia la construcción de un derecho energético ambiental como disciplina autónoma. Jurídicas CUC, 12(1), 177-204. http://dx.doi.org/10. 17981/juridcuc.12.1.2016.10

Pérez, G. (2002). Investigación cualitativa. Retos e interrogantes. II técnicas y análisis de datos. Madrid: La Muralla.

Pérez-Acosta, A. y Urrá, M. (2015). El Premio Rubén Ardila a la investigación científica en psicología (2005-2015): De la psicología organizada a la historia de las ciencias del comportamiento. Cultura Educación y Sociedad, 6(2), 77-89. Recuperado de https://revistascientificas.cuc.edu.co/index.php/culturaeducacionysociedad/article/view/1042.

Rodriguez, L. (2012). Teoría y Técnica de Entrevista. Recuperado de http://www. aliat.org. $\mathrm{mx} /$ BibliotecasDigitales/salud/Teoria_y_tecnica_de_la_entrevista. pd.

Schutter, A. (1987). Metodo y proceso de la investigación participativa en la capacitación rural. Patzcuaro: CREFAL.

UNESCO. (1975). Carta de Belgrado. (Línea de tiempo ambiental timeline. Timetoast. Recuperado de https://www. timetoast.com/timelines/linea-de-tiemp o-a mbiental-4c 54c 7 a 7 - 00 e 8 4055-b721-273549a7cf4e 